\title{
Kottman's constant, packing constant and Riesz angle in some classes of Köthe sequence spaces
}

\section{BOYAN ZLATANOV}

\section{ABSTRACT.}

We have found a sufficient condition in order that the Kottman constant to be equal to the Riesz angle for Köthe sequence spaces. We have found the ball packing constant in weighted Orlicz sequence spaces, endowed with Luxemburg or $p$-Amemiya norm. We have calculated the Riesz angle for Musielak-Orlicz, Nakano, weighted Orlicz, Orlicz, Orlicz-Lorentz, Lorentz and Cesaro sequence spaces.

\section{REFERENCES}

[1] Blasco, O. and Gregori, P., Type and cotype in vector-valued Nakano sequence spaces, J. Math. Anal. Appl., 264 (2001), No. 2, 657-672

[2] Borwein, J. and Sims, B., Non-expansive mappings on Banach lattices and related topics, Houston J. Math., 10 (1984), No. 3, 339-356

[3] Burlack, J. A. C., Rankin, R. A. and Robertson, A. P., The packing of spheres in the spaces $l_{p}$, Glasg. Math. J., 4 (1958), No. 1, 22-25

[4] Cerdà, J., Hudzik, H., Kamińska, A. and Mastyło, M., Geometric properties of symmetric spaces with applications to Orlicz-Lorentz spaces, Positivity, 2 (1998), No. 4, 311-337

[5] Changsen, Y. and Fenghui. W., On a generalized modulus of convexity and uniform Normal structure, Acta Mathematica Scientia, 27B (2007), No. 4, 838--844

[6] Chen, S., Geometry of Orlicz Spaces [PhD thesis], Warszawa, Polish Academy of Sciences 1996

[7] Clarkson, J. A. Uniformly convex spaces, Trans. Amer. Math. Soc., 40 (1936), No. 3, 394-414

[8] Cui, Y., Duan, L., Hudzik, H. and Wisła, M., Basic theory of $p$-Amemiya norm in Orlicz spaces $(1 \leq p \leq \infty)$ : Extreme points and rotundity in Orlicz spaces endowed with these norms, Nonlinear Anal., 69 (2008), No 5-6, 1796-1816

[9] Cui, Y. and Hudzik, H., On the uniform Opial property in some modular sequence spaces, Funct. Approximatio, Comment. Math., 26 (1998), 93-102

[10] Cui, Y. and Hudzik, H., Packing constant for Cesàro sequence spaces, Nonlinear Anal., 47 (2001), No. 4, 2695-2702

[11] Cui, Y., Hudzik, H. and Wisła, T., M-constants, Dominguez-Benavides coefficient, and weak fixed point property in Orlicz sequence spaces equipped with the p-Amemiya norm, Fixed Point Theory Appl., 2016:89 (2016)

[12] Cui, Y., Hudzik, H. and Zhang, T., On some geometric properties of certain Köthe sequence spaces, Math. Bohem., 124 (1999), No. 2-3, 303-314

[13] Delpech, S., Asymptotic uniform moduli and Kottman constant of Orlicz sequence spaces, Rev. Mat. Complut., 22 (2009), No. 2, 455-467

[14] Diestel, J., Sequences and Series in Banach Spaces, Springer-Verlag, New York, 1984

[15] Hernandez, F., On the galb of weighted Orlicz sequence spaces II, Arch. Math. (Basel), 45 (1985), No. 2, 158-168

Received: 12.01.2018; In revised form: 30.05.2018; Accepted: 07.06.2018

2010 Mathematics Subject Classification. 46B20, 46E30, 46B45, 46A45.

Key words and phrases. Köthe sequence spaces, Musielak-Orlicz sequence space, weighted Orlicz sequence space, Luxemburg norm, Amemiya norm, Kottman's constant, Riesz angle, Packing constant. 
[16] He, X., Yu, J., Cui, Y. and Huo, X. Packing constant in Orlicz sequence spaces equipped with the p-Amemiya norm, Abstr. Appl. Anal., 2014 (2014), Article ID 626491, 7 pages. http://dx.doi.org/10.1155/2014/626491.

[17] Hudzik, H., Every nonreflexive Banach lattice has the packing constant equal to 1/2, Collect. Math., 44 (1993), No. 1-3, 129-134

[18] Hudzik, H., Wu, C. X. and Ye, Y. N., Packing constant in Musielak-Orlicz sequence spaces equipped with the Luxemburg norm, Revista Matemática de la Universidad Complutense de Madrid, 7 (1994), No. 1, 13-26

[19] James, R. C., Uniformly non-square Banach spaces, Ann. of Math. (2), 80 (1964), No. 3, 542-550

[20] Kamińska, A., On comparison of Orlicz spaces and Orlicz classes, Funct. Approximatio, Comment. Math., 11 (1981), 113-125

[21] Kamińska, A., Rotundity of Orlicz-Musielak sequence spaces, Bull. Acad. Pol. Sci., Sér. Sci. Math., 29 (1981), 137-144

[22] Kamińska, A., On Uniform convexity of Orlicz spaces, Indag. Math., 44 (1982), No. 1, 27-36

[23] Kamińska, A., Flat Orlicz-Musielak sequence spaces, Bull. Acad. Pol. Sci., Sér. Sci. Math., 30 (1982), 347-352

[24] Kamińska, A., Uniform rotundity of Musielak-Orlicz sequence spaces, J. Approx. Theory, 47 (1986), No. 4, 302-322

[25] Kamińska, A., Some remarks on Orlicz-Lorentz spaces, Math. Nachr., 147 (1990), No. 1, 29-38

[26] Kamińska, A. and Lee, H. J. Banach-Saks properties of Musielak-Orlicz and Nakano sequence spaces, Proc. Amer. Math. Soc., 142 (2014), No. 2, 547-558

[27] Kamińska, A. and Mastylo. M., The Schur and (weak) Dunford-Pettis property in Banach lattices, J. Aust. Math. Soc., 73 (2002), No. 2, 251-278

[28] Kottman, C. A., Packing and reflexivity in Banach spaces, Trans. Amer. Math. Soc., 150 (1970), No. 2, 565-576

[29] Kottman, C. A., Subsets of the unit ball that are separated by more than one, Studia Math., 53 (1975), No. 1, 15-27

[30] Lin, P. K. and Sun, H. Y., Some geometric properties of Lorentz-Orlicz spaces, Arch. Math., 64 (1995), No. 6, 500-511

[31] Lindenstrauss, J., On the modulus of smoothness and divergent series in Banach spaces, Michigan Math. J. 10 (1963), 241-252

[32] Lindenstrauss, J. and Tzafriri, L., Classical Banach Spaces I, Springer-Verlag, Berlin, 1977

[33] Lindenstrauss, J. and Tzafriri, L., Classical Banach Spaces II, Springer-Verlag, Berlin, 1979

[34] Maleev, R. and Zlatanov. B., Smoothness in Musielak-Orlicz sequence spaces, C. R. Acad. Bulgare Sci., 55 (2002), No. 6, 11-16

[35] Maluta, E. and Papini, P. L., Estimates for Kottman's separation constant in reflexive Banach spaces, Colloq. Math., 117 (2009), No. 1, 105-119

[36] Matuszewska, W. and Orlicz, W. On certain properties of $\varphi$-functions, Bull. Acad. Polon. Sci. Sér. Sci. Math. Astronom. Phys., Phys., 8 (1960), 439-443

[37] Maurey, B., Points fixes des contractions de certains faiblement compacts de $L^{1}$, Séminaire Analyse Fonctionnelle (dit "Maurey-Schwartz") 1980-1981, Exposé $N^{\circ}$ VIII, 1-18

[38] Musielak, J., Orlicz Spaces and Modular Spaces, Lecture Notes in Math., Springer-Verlag, Berlin, 1983

[39] Nezir, V., Fixed Point Property for $c_{0}$-Like Spaces [PhD thesis], University of Pittsburgh 2012

[40] Rankin, R. A., The closest packing of spherical caps in $n$ dimensions, Glasg. Math. J., 2 (1955), No. 3, 139-144

[41] Rankin, R. A., On packing of spheres in Hilbert space, Glasg. Math. J., 2 (1955), No. 3, 145-146

[42] Rao, M. and Ren, Z., Applications of Orlicz Spaces, Pure and Applied Mathematics, Marcel Dekker Inc., New York, 2002

[43] Shi, Z. and Wang, T., On the packing spheres of some sequence spaces, Analysis, 17 (1997), No. 4, 379-386

[44] Sudsukh, C. and Mongkolkeha, C., Some geometric properties of generalized modular sequence spaces defined by Zweier operator, J. Nonlinear Sci. Appl., 9 (2016), 2289-2297

[45] Webb, J. R. L. and Zhao, W.,On connections between set and ball measures of noncompactness, Bull. Lond. Math. Soc., 22 (1990), No. 5, 471-477

[46] Well, J. H. and Williams, L. R., Embedding and Extension Problems in Analysis, Lecture Notes in Math., Springer-Verlag, New York, 1975

[47] Woo, J., On modular sequence spaces, Studia Math., 48 (1973), No. 3, 271-289

[48] Xisheng Yu, The packing spheres constant for a class of separable Orlicz function spaces, Journal of Mathematics Research, 1 (2009), No. 2, 21-27

[49] Yan, Y., On some expressions for the Riesz angle of Orlicz sequence spaces, Sib. Math. J., 44 (2003), No. 4, 739-747

[50] Yan, Y., Packing constants in Orlicz-Lorentz sequence spaces, Taiwanese J. Math., 15 (2011), No. 6, 2403-2428 
[51] Zlatanov, B., On Weak uniform normal structure in weighted Orlicz sequence spaces, J. Math. Anal. Appl., 341 (2008), No 2, 1042-1054

[52] Zlatanov, B., On a class of Köthe sequence spaces with normal structure, Acta Math. Sci., 31B (2011), No 2, 576-590

[53] Zlatanov, B., Some expressions for the Riesz angle of weighted Orlicz sequence spaces, Math. Sci., (2013), 7:13, doi:10.1186/2251-7456-7-13

Plovdiv University "Paisi Hilendarski"

FACULTY OF MATHEMATICS AND INFORMATICS

TSAR ASSEN 24, 4000, Plovdiv, BUlgaria

Email address: bzlatanov@gmail.com 